\title{
Correction to: Epilepsy related multimorbidity, polypharmacy and risks in adults with intellectual disabilities: a national study
}

\author{
James J. Sun ${ }^{1} \cdot$ Bhathika Perera ${ }^{1} \cdot$ William Henley ${ }^{2} \cdot$ Heather Angus-Leppan ${ }^{3} \cdot$ Indermeet Sawhney $^{4} \cdot$ Lance Watkins $^{5}$. \\ Kiran N. Purandare ${ }^{6} \cdot$ Mogbeyiteren Eyeoyibo $^{7} \cdot$ Mark Scheepers $^{8} \cdot$ Geraldine Lines $^{9} \cdot$ Robert Winterhalder $^{9}$. \\ Samantha Ashby ${ }^{10} \cdot$ Ravindu De Silva $^{1}$. Jonjo Miller ${ }^{1}$. David E. Philpott ${ }^{11}$. Chris Ashwin ${ }^{11}$. Joshua Howkins ${ }^{11}$.

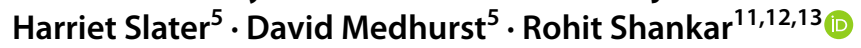

Published online: 5 March 2022

(c) The Author(s), under exclusive licence to Springer-Verlag GmbH Germany 2022

\section{Correction to: Journal of Neurology} https://doi.org/10.1007/s00415-021-10938-3

The original version of this article unfortunately contained a mistake. Author name Ravindu De Silva was incorrectly written as Ravi De Silva.

The original article can be found online at https://doi.org/10.1007/ s00415-021-10938-3.

Rohit Shankar

Rohit.shankar@plymouth.ac.uk

1 Barnet, Enfield and Haringey Mental Health NHS Trust, London, UK

2 University of Exeter Medical School, Exeter, UK

3 Royal Free London NHS Foundation Trust, London, UK

4 Hertfordshire Partnership University NHS Foundation Trust, St Albans, UK

5 Swansea Bay University Health Board, Swansea, UK

6 Central and North West London NHS Foundation Trust, London, UK

7 Kent and Medway NHS and Social Care Partnership Trust, Kent, UK
Gloucestershire Health and Care NHS Foundation Trust, Gloucester, UK

9 Oxleas NHS Foundation Trust, London, UK

10 SUDEP Action, Wantage, UK

11 Cornwall Partnership NHS Foundation Trust, Truro TR4 9LD, UK

12 University of Plymouth Peninsula School of Medicine, Plymouth, UK

13 Cornwall Intellectual Disability Equitable Research (CIDER), Threemilestone Industrial Estate, Cornwall TR4 9LD, Truro, UK 